\title{
Detection of high levels of mutations involved in anti-malarial drug resistance in Plasmodium falciparum and Plasmodium vivax at a rural hospital in southern Ethiopia
}

Patricia Mula* ${ }^{1 *}$ Amalia Fernández-Martínez', Aida de Lucio', Jose Manuel Ramos²,3 Francisco Reyes², Vicenta González ${ }^{1}$, Agustín Benito ${ }^{1}$ and Pedro Berzosa ${ }^{1}$

\begin{abstract}
Background: In Ethiopia, malaria is caused by Plasmodium falciparum and Plasmodium vivax, and anti-malarial drug resistance is the most pressing problem confronting control of the disease. Since co-infection by both species of parasite is common and sulphadoxine-pyrimethamine (SP) has been intensively used, resistance to these drugs has appeared in both $P$. falciparum and $P$. vivax populations. This study was conducted to assess the prevalence of anti-malarial drug resistance in $P$. falciparum and $P$. vivax isolates collected at a rural hospital in southern Ethiopia.

Methods: A total of 1,147 patients with suspected malaria were studied in different months across the period 2007-2009. Plasmodium falciparum dhfr and dhps mutations and P. vivax dhfr polymorphisms associated with resistance to SP, as well as $P$. falciparum pfcrt and pfmdr1 mutations conferring chloroquine resistance, were assessed.

Results: PCR-based diagnosis showed that 125 of the 1147 patients had malaria. Of these, $52.8 \%$ and $37.6 \%$ of cases were due to $P$. falciparum and $P$. vivax respectively. A total of 10 cases (8\%) showed co-infection by both species and two cases (1.6\%) were infected by Plasmodium ovale. Pfdhfr triple mutation and pfdhfr/pfdhps quintuple mutation occurred in 90.8\% (95\% confidence interval [Cl]: $82.2 \%-95.5 \%$ ) and $82.9 \%$ (95\% Cl: $72.9 \%-89.7 \%$ ) of $P$. falciparum isolates, respectively. Pfcrt T76 was observed in all cases and pfmdr1 Y86 and pfmdr1 Y1246 in 32.9\% (95\% Cl: $23.4 \%-44.15 \%)$ and $17.1 \%$ (95\% Cl: 10.3-27.1\%), respectively. The P. vivax dhfr core mutations, N117 and R58, were present in 98.2\% (95\% Cl: 89.4-99.9\%) and 91.2\% (95\% Cl: 80.0-96.7\%), respectively.

Conclusion: Current molecular data show an extraordinarily high frequency of drug-resistance mutations in both $P$. falciparum and P. vivax in southern Ethiopia. Urgent surveillance of the emergence and spread of resistance is thus called for. The level of resistance indicates the need for implementation of entire population access to the new first-line treatment with artemether-lumefantrine, accompanied by government monitoring to prevent the emergence of resistance to this treatment.
\end{abstract}

\footnotetext{
* Correspondence: pmula@isciii.es

'National Centre for Tropical Medicine, Institute of Health Carlos III. C/Sinesio Delgado 4-6, 28029, Madrid, Spain

Full list of author information is available at the end of the article
}

(c) 2011 Mula et al; licensee BioMed Central Ltd. This is an Open Access article distributed under the terms of the Creative Commons 


\section{Background}

Malaria is the vector-borne disease with the highest impact on the world's human population. In 2008, there were an estimated 243 million cases, leading to nearly 863,000 malaria-related deaths [1]. Although malariaendemic areas are mainly restricted to tropical and subtropical regions at present, several models nonetheless project the geographical expansion of potential malaria transmission over the next few decades, along with more substantial changes later in the century [2]. Approximately $90 \%$ of clinically manifest infections of and practically all deaths from malaria are caused by Plasmodium falciparum. Ranking second in importance is Plasmodium vivax, which accounts for nearly $10 \%$ of global malaria incidence. Roughly $90 \%$ of all malaria cases occur in tropical Africa [3].

Approximately 52 million people in Ethiopia are considered to be at risk of the disease [4]. While 4 to 6 million clinical malaria cases are annually reported by the country's health facilities, the real number is estimated to be as high as 10 to 15 million [5]. The major Plasmodium species causing malaria in Ethiopia are $P$. falciparum (about $60 \%$ of cases) and $P$. vivax (about $40 \%$ of cases), with the former being the cause of the most severe clinical manifestations and most deaths [6-8]. Malaria transmission follows a seasonal pattern (September-November), depending on the altitude and rainy season [7,9]. Epidemic malaria is frequent [10], particularly in the highlands (1,000-2,000 $\mathrm{m}$ above sea level).

Anti-malarial drug resistance in P. falciparum and $P$. vivax is the most pressing problem confronting malaria control in many endemic countries [11,12]. Plasmodium falciparum has developed resistance to a series of drugs, and $P$. vivax is resistant to chloroquine (CQ) and not primaquine-tolerant (PQ) $[13,14]$. Furthermore, in countries where intensive use has been made of sulphadoxine-pyrimethamine (SP), resistance to these drugs has appeared in $P$. vivax populations [15-20], though some authors view this as being a sign of innate resistance to sulphadoxine in such parasites [21].

Current studies report high rates of therapeutic failure (72\%) in some areas of Ethiopia [22], due to the presence of SP resistance in the two principal species. In 2004, artemether-lumefantrine (AL) $\left(\right.$ Coartem $\left.^{\circledR}\right)$ was introduced into the country as a first-line treatment, and the national guidelines have since prescribed this drug for uncomplicated malaria [4]. Even so, not everyone is benefiting from this treatment because $85 \%$ of the population live in rural areas where access to basic health care is severely limited.

In general, the presence of high resistance to SP has stopped it from being used as a treatment in African endemic countries, and so nowadays it is only used, in combination with insecticide-treated mosquito nets, for Intermittent Preventive Treatment (IPT) in pregnant women and in areas where it has been given to children under five years of age has provided encouraging results [23].

The study of point mutations (Single Nucleotide Polymorphisms - SNPs) in molecular markers is an extremely useful epidemiological tool, which enables the emergence and spread of mutant parasites to be monitored. Analyses of different molecular markers of resistance are currently used, namely: the T76 point mutation in the P. falciparum pfcrt gene; the Y86, F184, C1034, N1042 and Y1246 point mutations in the pfmdr1 gene [24]; the L164, N/T108, I51 and R59 point mutations in the pfdhfr gene; and the G437 and G581, A/F436, E540 and S613 point mutations in the pfdhps gene [12,24-28].

Plasmodium vivax resistance to SP is associated with mutations that appear in the homologous genes, $p v d h f r$ (N117, R58 and T117) and pvdhps (G383, G553) $[17,18]$. Mutations in $p v d h f r$ have been implicated in resistance to pyrimethamine in vivo [29]. Furthermore, it seems that pvdhfr mutations at residues 117 and 58 have been observed to arise first when drug pressure is applied $[17,18,30]$. The double and triple mutations, N117/R58 and N117/R58/L57, are associated with delayed parasite clearance following SP treatment [17] and with therapeutic failure in many regions of southeast Asia [17,31,32], Iran [30], Pakistan [33], India [34], Colombia [28] and Madagascar [35].

However, the appearance of different combinations of L57/R58/N117/G383/G553 mutations in both the pvdhfr and pvdhps genes has been associated with SP treatment failure only in patients infected by $P$. vivax and not in those co-infected by both species $[36,37]$ in which no mutations have been found in the orthologous genes of P. falciparum.

This study sought to determine the following: the prevalence of $P$. falciparum and $P$. vivax in patients with clinically suspected malaria, attending the Gambo General Rural Hospital in Ethiopia; and the prevalence of mutations in genes involved in resistance to different anti-malarial drugs, based on samples collected in different months across the period 2007-2009.

\section{Methods}

\section{Study site and biological samples}

The Gambo General Rural Hospital is situated in the province of West Arsi, Ethiopia, 245 kilometres southeast of the capital, Addis Ababa (Figure 1), at an altitude of 2,250 metres (approximately 7,382 feet) above sea level. The hospital provides daily health care and covers nine peripheral health centres serving 23 villages, with a 

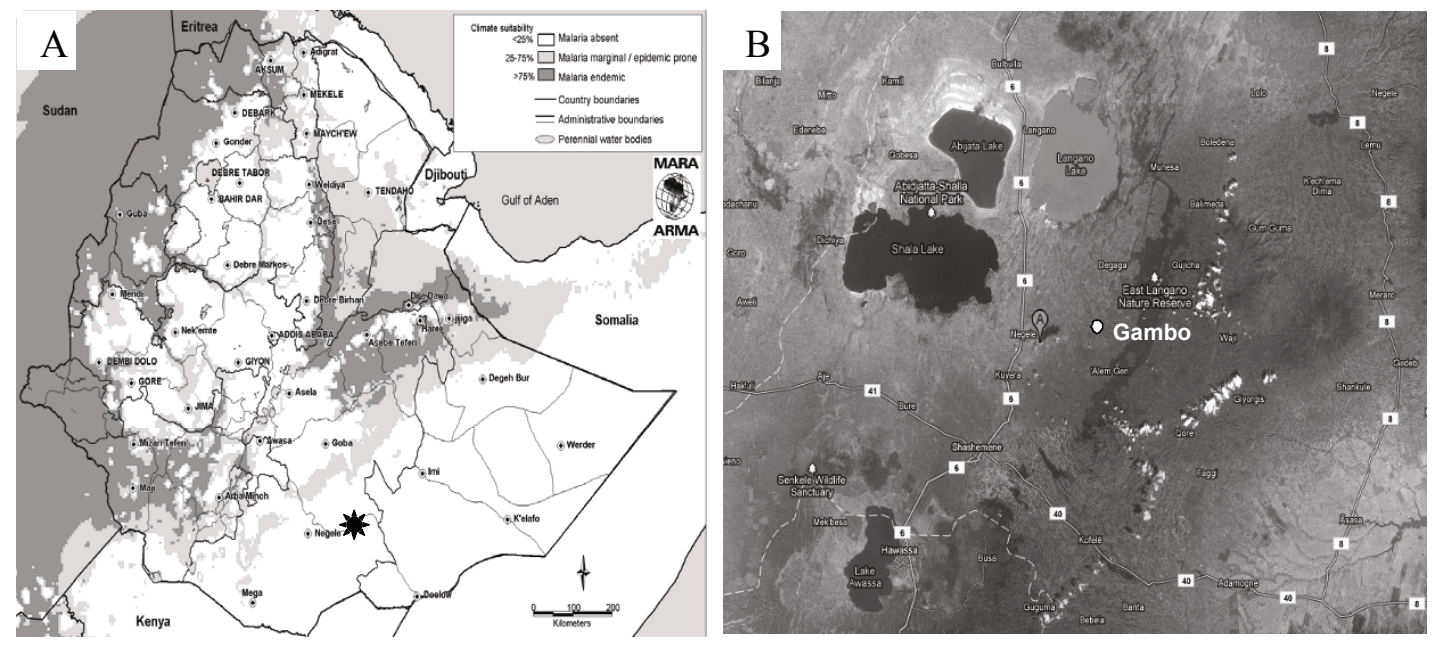

Figure 1 Gambo location in Ethiopia. The left figure $(1 \mathrm{~A})$ displays the location of Gambo on the map of Ethiopia (black star). The right figure (1 B) shows the satellite image of Gambo. Source of maps: 1 A: http://www.mara.org.za/; 1 B: Google Maps.

total population of 98,000. Patients may come from bordering areas, lying at lower altitudes.

The study was conducted during different calendar months across the period 2007-2009. At the hospital, a total of 1,147 blood samples were collected from patients (age range, 1 month to 75 years) with clinically suspected malaria (see Table 1). Two capillary blood specimens were taken from each patient, one for confirmation of Plasmodium infection by microscopic observation of thin and thick Giemsa-stained blood films, and the other on filter paper (Whatman ${ }^{\odot} 3 \mathrm{MM}$ ) for molecular assay.

DNA extraction was performed using commercial kits (Speedtools tissue DNA Extraction Kit, Biotools), and Plasmodium species were subsequently confirmed by Seminested-Multiplex PCR [38]. The nested PCR-RFLP technique was used to analyse: codons 76 of $p f c r t, 86$ and 1246 of pfmdr1, 51, 59, 108 and 164 of pfdhfr and 436, 437, 540 and 581 of pfdhps from samples infected by P. falciparum [39]; and codons pvdhfr 57, 58 and 117 and pvdhps 383 and 553 from samples infected with $P$. vivax. The laboratory conditions and restriction enzymes have been described elsewhere $[17,18,30]$. As in other resistance studies [40], genes that showed mixed wildtype and mutant alleles were deemed to be mutant only

Table 1 Number of samples collected by year of study.

\begin{tabular}{cccc}
\hline Year & Months & No of Months & Samples \\
\hline 2007 & Nov-Dec & 2 & 175 \\
2008 & Jan-May & 5 & 432 \\
2009 & Feb-May & 4 & 540 \\
\hline & & Total months: 11 & Total samples: 1,147
\end{tabular}

(Nov: November/Dec: December/Jan: January/Feb: February). when the amplification fragment was more intense for the latter. The study protocol was approved by the research review boards of the Institute of Health Carlos III (Madrid, Spain) and Gambo General Rural Hospital. Study participants with microscopy-confirmed malaria infection were treated in accordance with Ethiopian national guidelines $[4,5]$. A descriptive analysis was performed and estimates of prevalence along with their 95\% confidence intervals (CIs) were obtained, using the method described by Newcombe [41].

\section{Results}

\section{Molecular results}

The presence of parasites was confirmed by SeminestedMultiplex-PCR in all the samples. Of the 1,147 samples, a total of 125 (10.9\%; 95\% CI: 9.2-12.8\%) were shown to be infected by Plasmodium sp., which broke down as follows: P. falciparum, 66 (5.8\%; 95\% CI: 4.5-7.3\%); P. vivax, 47 (4.1\%; 95\% CI: 3.1-5.5\%); P. falciparum and $P$. vivax combined, 10 (0.9\%; 95\% CI: 0.4-1.6\%); and Plasmodium ovale, 2 (0.2\%; 95\% CI: $0.03-0.7 \%)$. The prevalence of Plasmodium species in infected cases $(\mathrm{n}=125)$ is shown in Table 2.

The frequencies of mutations and the combinations of these in the genes involved in anti-malarial drug

Table 2 Prevalence of Plasmodium species in infected patients $(n=125)$.

\begin{tabular}{ccc}
\hline Infection & $\mathbf{n}=\mathbf{1 2 5}$ & Frequency (\%) \\
\hline P. falciparum & 66 & $52.8 \%$ \\
P. vivax & 47 & $37.6 \%$ \\
P. ovale & 2 & $1.6 \%$ \\
Mixta P. falciparum/P. vivax & 10 & $8.0 \%$ \\
\hline
\end{tabular}


Table 3 Prevalence of mutations conferring resistance to chloroquine and sulphadoxine-pyrimethamine in Plasmodium falciparum $(n=76)$.

\begin{tabular}{lcc}
\hline Gene Locus & $\mathbf{n}=\mathbf{7 6}$ & Mutation (\%) \\
\hline pfcrt 76 & 76 & $(100 \%)$ \\
pfmdr1 86 & 25 & $(32.9 \%)$ \\
pfmdr1 1246 & 13 & $(17.1 \%)$ \\
pfdhfr 51 & 74 & $(97.4 \%)$ \\
pfdhfr 59 & 69 & $(90.8 \%)$ \\
pfdhfr 108 & 76 & $(100 \%)$ \\
pfdhfr 164 & 1 & $(1.3 \%)$ \\
pfdhps 436 & 14 & $(18.4 \%)$ \\
pfdhps 437 & 70 & $(92.1 \%)$ \\
pfdhps 540 & 52 & $(68.4 \%)$ \\
pfdhps 581 & 11 & $(14.5 \%)$ \\
\hline
\end{tabular}

resistance in P. falciparum are shown in Table 3 and Figure 2 respectively. Whereas all samples infected with P. falciparum presented with the pfcrt T76 mutation (95 CI: $95.1-100 \%), 32.9 \%$ (95\% CI: 23.4-44.1\%) and $17.1 \%$ (95\% CI: 10.3-27.1\%) of samples infected with P. falciparum had the pfmdr1 Y86 and pfmdr1 Y1246 mutations respectively.

As with pfcrt T76, all samples infected with $P$. falciparum showed a $100 \%$ prevalence of mutation at pfdhfr N108. Only one case (1.3\%; 95\% CI: 0.2-7.1\%), however, presented with the mutation at pfdhfr L164. The $p f d h f r$ I51 and R59 mutations, responsible for modulating resistance to pyrimethamine, appeared in $97.4 \%$ (95\% CI: $90.9-99.3 \%)$ and $90.8 \%$ (95\% CI: 82.2-95.5\%) of cases respectively. The triple I51/R59/N108 mutation was observed in over 90\% (95\% CI: 82.2-95.5\%) of cases (Figure 2).

Insofar as the SNPs in pfdhps were concerned, none of the samples had wild genotypes at the codons studied. Both pfdhps A436 and pfdhps G581 mutations appeared at low frequencies $(18.4 \%$ [95\% CI: $11.3-28.9 \%$ ] and $14.5 \%$, [95\% CI: 8.3-24.1\%] respectively) compared to the pfdhps G437 and pfdhps E540 mutations, which appeared in 92.1\% (95\% CI: 83.8-96.3\%) and 68.4\% (95\% CI: $57.3-77.8 \%)$ of cases respectively. The pfdhps double mutation, G437/E540, appeared in $84.2 \%$ (95\% CI: 74.4$90.7 \%)$ of cases.

The $p f d h f r / p f d h p s$ quadruple and quintuple mutations, N108/I51/R59+G437 and N108/I51/R59+G437/E540 (both associated with therapeutic failure of SP), displayed a prevalence of over $80 \%$ (95\% CI: 70-87.7\%) among cases infected by P. falciparum (Figure 2).

While $98.2 \%$ (95\% CI: 89.4-99.9\%) of samples infected with $P$. vivax showed the N117 mutation at codon 117 of the pvdhfr gene, R58 appeared at codon 58 in $91.2 \%$ (95\% CI: $80.0-96.7 \%)$ of cases. The pvdhfr double mutation, N117/R58, was observed in most cases of $P$. vivax infection (91.2\%; 95\% CI: 80.0-96.7\%) but no case displayed mutation at codon 57 (95\% CI: 0-7.9\%).

With respect to the $p v d h p s$ gene, mutation was found at codon 553, with a prevalence of $3.5 \%$ (95\% CI: 0.6$13.2 \%)$ and appeared at the same frequency (3.5\%; (95\% CI: $0.6-13.2 \%)$ in combination with the double N117/ R58 mutation (Table 4).

All cases that were co-infected by both species $(\mathrm{n}=$ 10) and presented with mutations in pfdhfr/pfdhps (I51/ R59/N108, 80\% [95\% CI: 49.0-94.3\%]; N108/I51/R59

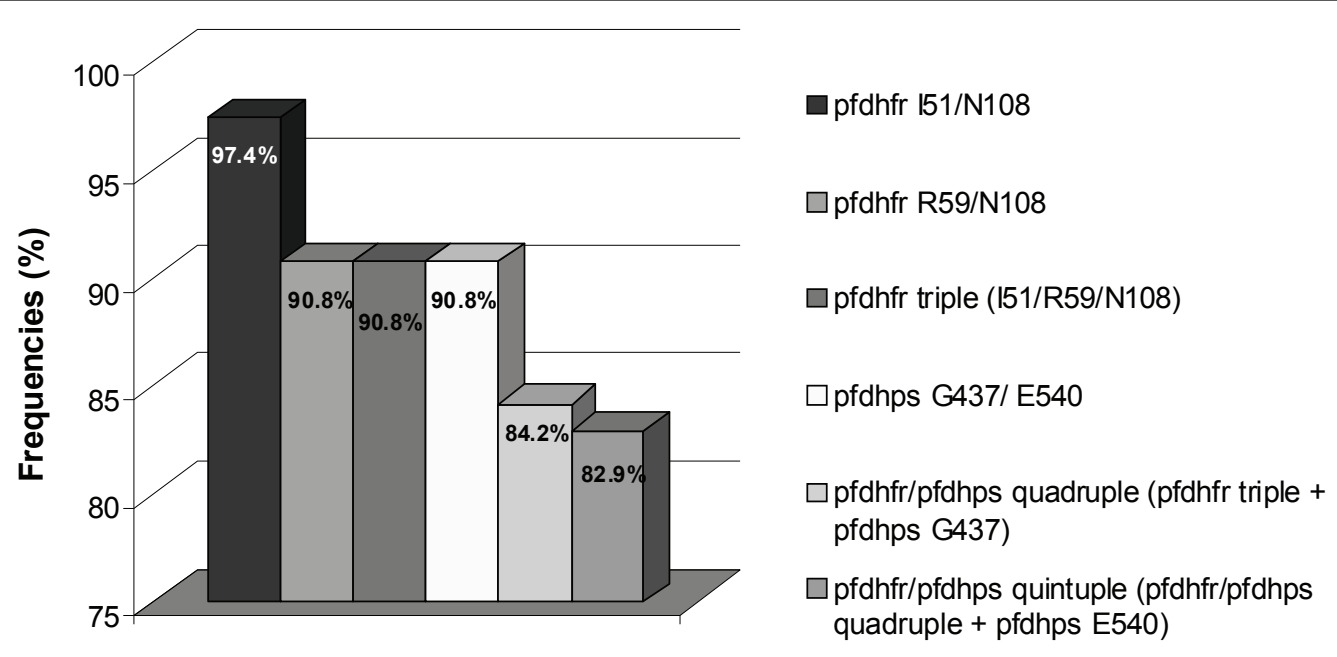

Grouped pfdhfr/pfdhps alleles

Figure 2 Prevalence of grouped $p f d h f r / p d h p s$ mutant alleles in $P$. falciparum linked to sulphadoxine-pyrimethamine treatment failure $(n=76)$. 
Table 4 Prevalence of mutations conferring resistance to sulphadoxine-pyrimethamine in Plasmodium vivax ( $n=57$ ).

\begin{tabular}{lcc}
\hline Gene Locus & $\mathbf{N}=\mathbf{5 7}$ & Mutation (\%) \\
\hline pvdhfr R58 & 52 & $(91.2 \%)$ \\
pvdhfr L57 & 0 & $(0 \%)$ \\
pvdhfr N117 & 56 & $(98.2 \%)$ \\
pvdhfr T117 & 0 & $(0 \%)$ \\
pvdhfr R58/N117 & 52 & $(91.2 \%)$ \\
pvdhfr R58/N117/L57 & 0 & $(0 \%)$ \\
pvdhps G383 & 0 & $(0 \%)$ \\
pvdhps G553 & 2 & $(3.5 \%)$ \\
pvdhfr/pvdhps R58/N117/G553 & 2 & $(3.5 \%)$ \\
\hline
\end{tabular}

+G437, 80\% [95\% CI: 49.0-94.3\%]; and N108/I51/R59 +G437/E540, 70\% [95CI: 39.7-89.2]), also introduced the double mutation, N117/R58, (100\%; 95\% CI: 72.3-100\%) in $p v d h f r$.

\section{Discussion}

In areas where $P$. falciparum and $P$. vivax co-exist, parasite-specific diagnosis and choice of effective treatment is crucial to prevent the emergence and spread of resistance. In malaria-endemic areas, the occurrence of $P$. falciparum $/ P$. vivax co-infection is frequent and it is, therefore, common for $P$. vivax to have been exposed to treatment with SP.

In Ethiopia, not only are there wide interregional differences in the endemicity and transmission of malaria, but there is also a significant lack of information on the effectiveness of anti-malarial drugs. In 1999, the CQ treatment failure rate in the first two weeks still stood at $88 \%$ in the centre of the country [42]. In 2005, SP therapeutic failure within two to four weeks of follow-up was $36 \%$ and $72 \%$ respectively. In this same year, however, treatment with AL yielded an appropriate clinical and parasitological response of 99\% [22].

With regard to the prevalence of species, a decrease of $P$. falciparum mono-infection and an increase of $P$. vivax mono-infection was observed in contrast to other results obtained by other studies previously conducted in Ethiopia $[6,7,43,44]$. The predominant species was $P$. falciparum, which appeared in approximately 53\% of cases, followed by $P$. vivax with a prevalence higher than $30 \%$, and a small proportion cases of co-infection by both species. In addition, two cases of infection by Plasmodium ovale were detected. This finding is rather singular; in that $P$. ovale has never been previously described in Ethiopia, and might be accounted for by the migration process from the western side of the continent to this country, or people returning after having gone to work in other areas of Africa with prevalence of $P$. ovale. In the future, more studies about the prevalence of malaria species should be performed in Ethiopia, to know the factors are influencing in these changes of prevalence.

As with other studies in which high rates of therapeutic failure of CQ and SP have been detected, our results likewise show a high prevalence of these mutations linked to resistance in $P$. falciparum, particularly T76 in pfcrt (responsible for resistance to CQ) and the triple mutation in $p f d h f r$ (responsible for treatment failure in SP) (see Table 3 and Figure 2). Furthermore, the high rate of the pfdhps double mutation, G437/E540, responsible for conferring a high degree of resistance to sulphadoxine and for therapeutic failure to SP in the presence of the triple mutation in $p f d h f r$, has resulted in the $p f d h f r / p f d h p s$ quintuple mutation being present in over $80 \%$ of cases. This corresponds to the low SP-treatment efficacy rates registered in different regions of Ethiopia [22] and confirms the need for AL to be made available countrywide as the first-line treatment.

The pfcrt T76, pfmdr1 Y86 and pfmdr1 Y1246 mutations are very useful molecular markers of CQ resistance in areas where resistance rates are low to mild $[45,46]$. This study showed high to moderate prevalence of $p f c r t$ and $p f m d r 1$ mutations, respectively, to be consistent with low CQ efficacy in Ethiopia [42].

In the case of $P$. vivax, there was a high prevalence of the $p v d h f r$ R58 and pvdhfr N117 mutations. Furthermore, the $p v d h f r$ double mutation, N117/R58, appeared in 52 out of 57 cases infected by $P$. vivax (taking the cases of $P$. falciparum co-infection into account), and 42 out of 47 cases infected by this species alone. The pvdhps G553 mutation always appeared in combination with pvdhfr double mutation R58/N117, resulting pvdhfr/pvdhps R58/N117/G553 mutation in two cases of $P$. vivax mono-infection. This finding could explain a possible SP treatment failure in these patients, as has been associated in other studies [36,37]. Yet $p v d h f r$ T117, L57, $p v d h f r$ triple or $p v d h f r / p v d h p s$ quadruple mutations were not in evidence, in the last case neither in $P$. vivax mono-infection nor in $P$. vivax- $P$. falciparum co-infection. Although these multiple mutations seem to be necessary for in vivo resistance $[17,18,43]$, this finding nevertheless suggests widespread SP use, since the $p v d h f r$ mutations arise first under drug pressure $[17,18,43]$.

It should be taken into account that, when it comes to combined $P$. falciparum $/ P$. vivax infection, patients should be treated with a blood schizonticide (for both species) and a tissue schizonticide (for hepatic hypnozoites of $P$. vivax). In the past, SP was used as treatment for $P$. falciparum. As a result, $P$. vivax came into contact with the same treatment when it was present in mixed infections, and mutations in different genes linked to SP resistance have thus appeared in this species. The 
mutations in the pvdhps gene would be equally accounted for, i.e., here, only the G553 mutation, implicated in resistance to sulphadoxine and corresponding to the G581 mutation in P. falciparum, displayed a very low prevalence and appeared in the presence of the pvdhfr double mutation, N117/R58. In contrast, the G383 mutation, which corresponds to G437 in P. falciparum, was observed in neither case. This strengthens the hypothesis that the asymmetric selection process of mutations observed in P. falciparum is also applicable to P. vivax [21].

Samples co-infected by these two species showed mutations in either $p f d h f r / p f d h p s$ or $p v d h f r$, which would explain why treatment for both these species of Plasmodium could fail in such patients.

In view of the genotype results obtained, it would be advisable for a study of therapeutic efficacy to be conducted in this area, to ascertain whether the genotypic are the same as the phenotypic data, i.e., whether the parity mutation-resistance is produced in vivo.

Moreover, it would be advisable for future research to verify whether there were mutations in genes (such as atp6) related with resistance to artemisinin derivatives, the use of which as an anti-malarial drug has spread in Africa. Artemisinin-based combination therapy (ACT) (for example, using Coartem ${ }^{\circledR}$ ) has come into use in Ethiopia, though it is proving difficult to implement in rural areas due to the continued use of drugs, such as SP and $\mathrm{CQ}$, in combination with other medication, a practice that continues to favour the expansion of resistance.

\section{Conclusions}

1. The prevalence of Plasmodium species is slightly different from the results obtained by other authors in previous studies conducted in Ethiopia, showing an increase of vivax malaria cases and the presence of $P$. ovale for the first time.

2. The prevalence of mutations linked to $C Q$ and SP resistance detected in $P$. falciparum and $P$. vivax in southern Ethiopia is higher than expected.

3. Implementation of permanent surveillance of resistance molecular markers as an epidemiological tool will enable health professionals and authorities to be alerted to the possible emergence and spread of resistance to new lines of treatment, such as ACT.

4. It is essential that the entire population be provided with access to new first-line treatment with ACT, which should be given under government supervision to avoid the drug being mismanaged and so prevent the emergence of any new resistances.

\section{Acknowledgements}

We would like to thank the laboratory staff of the Gambo General Rural Hospital for their assistance in collecting the data, and the nursing staff for their care of the patients, and to the Tropical Disease Cooperative Research Network (RICET). This study (Project Reference TRPY1400/09) was funded by the Institute of Health Carlos III.

\section{Author details}

${ }^{1}$ National Centre for Tropical Medicine, Institute of Health Carlos III. C/Sinesio Delgado 4-6, 28029, Madrid, Spain. ªmbo General Rural Hospital, Shashemane, Ethiopia. ${ }^{3}$ Infectious Diseases Unit, Hospital General Universitario de Elche, Alicante, Spain.

\section{Authors' contributions}

PM was involved in the molecular studies, interpretation of results and drafted the manuscript. AFM and VG helped to do molecular studies. AL helped to do molecular studies and carried out sample collection. JMR performed the statistical analysis and interpretation, and helped to draft the manuscript. FR helped to collect the samples in Gambo (Ethiopia). AB helped to draft the manuscript and PB conceived and funded the project, carried out sample collection, and drafted the manuscript. All authors read and approved the final manuscript.

\section{Competing interests}

The authors declare that they have no competing interests.

Received: 31 May 2011 Accepted: 2 August 2011

Published: 2 August 2011

\section{References}

1. WHO: World Malaria Report 2009. Geneva: World Health Organization; 2009.

2. McMichael AJ, Woodruff RE, Hales S: Climate change and human health: present and future risks. Lancet 2006, 367:859-869.

3. WHO: World Malaria Report 2008. Geneva: World Health Organization; 2008.

4. Federal Democratic Republic of Ethiopia MoH: Guideline for malaria epidemic prevention and control in Ethiopia. Addis Ababa, Ethiopia; 2004

5. Federal Democratic Republic of Ethiopia MoH: National Five Year Strategic Plan for Malaria Prevention and Control in Ethiopia. Addis Ababa, Ethiopia; 2006

6. Abeku TA, van Oortmarssen GJ, Borsboom G, De Vlas SJ, Habbema JD: Spatial and temporal variations of malaria epidemic risk in Ethiopia: factors involved and implications. Acta Trop 2003, 87:331-340.

7. Tulu AN: The ecology of health and disease in Ethiopia. Malaria.Edited by: Zein Ahmed Z, Kloos H. Westview Press; 1993:341-352.

8. Jima D: The 2003 malaria situation in Ethiopia. Proceedings of the Panel Discussion on the 2003 Malaria Epidemics in Ethiopia. Addis Ababa, Ethiopia; 2004

9. Deressa W, Ali A, Enqusellassie F: Self-treatment of malaria in rural communities, Butajira, southern Ethiopia. Bull World Health Organ 2003, 81:261-268.

10. Zhou G, Minakawa N, Githeko AK, Yan G: Association between climate variability and malaria epidemics in the East African highlands. Proc Natl Acad Sci USA 2004, 101:2375-2380.

11. Ngo T, Duraisingh M, Reed M, Hipgrave D, Biggs B, Cowman AF: Analysis of pfcrt, pfmdr1, dhfr, and dhps mutations and drug sensitivities in Plasmodium falciparum isolates from patients in Vietnam before and after treatment with artemisinin. Am J Trop Med Hyg 2003, 68:350-356.

12. Wernsdorfer WH, Noedl $\mathrm{H}$ : Molecular markers for drug resistance in malaria: use in treatment, diagnosis and epidemiology. Curr Opin Infect Dis 2003, 16:553-558.

13. Looareesuwan S, Buchachart K, Wilairatana P, Chalermrut K, Rattanapong Y, Amradee S, Siripiphat S, Chullawichit S, Thimasan K, Ittiverakul M, Triampon A, Walsh DS: Primaquine-tolerant vivax malaria in Thailand. Ann Trop Med Parasitol 1997, 91:939-943.

14. Murphy GS, Basri H, Purnomo, Andersen EM, Bangs MJ, Mount DL, Gorden J, Lal AA, Purwokusumo AR, Harjosuwarno S: Vivax malaria resistant to treatment and prophylaxis with chloroquine. Lancet 1993, 341:96-100.

15. Alam MT, Bora H, Bharti PK, Saifi MA, Das MK, Dev V, Kumar A, Singh N, Dash AP, Das B, Wajihullah, Sharma YD: Similar trends of pyrimethamine resistance-associated mutations in Plasmodium vivax and $P$. falciparum. Antimicrob Agents Chemother 2007, 51:857-863. 
16. Hastings MD, Porter KM, Maguire JD, Susanti I, Kania W, Bangs MJ, Sibley $\mathrm{CH}$, Baird JK: Dihydrofolate reductase mutations in Plasmodium vivax from Indonesia and therapeutic response to sulfadoxine plus pyrimethamine. J Infect Dis 2004, 189:744-750.

17. Imwong M, Pukrittakayamee S, Looareesuwan S, Pasvol G, Poirreiz J, White NJ, Snounou G: Association of genetic mutations in Plasmodium vivax dhfr with resistance to sulfadoxine-pyrimethamine: geographical and clinical correlates. Antimicrob Agents Chemother 2001, 45:3122-3127.

18. Imwong M, Pukrittayakamee S, Renia L, Letourneur F, Charlieu JP, Leartsakulpanich U, Looareesuwan S, White NJ, Snounou G: Novel point mutations in the dihydrofolate reductase gene of Plasmodium vivax: evidence for sequential selection by drug pressure. Antimicrob Agents Chemother 2003, 47:1514-1521.

19. de Pecoulas PE, Tahar R, Ouatas T, Mazabraud A, Basco LK: Sequence variations in the Plasmodium vivax dihydrofolate reductase-thymidylate synthase gene and their relationship with pyrimethamine resistance. $\mathrm{Mol}$ Biochem Parasitol 1998, 92:265-273.

20. Pukrittayakamee $S$, Chantra A, Simpson JA, Vanijanonta $S$, Clemens $R$, Looareesuwan S, White NJ: Therapeutic responses to different antimalarial drugs in vivax malaria. Antimicrob Agents Chemother 2000, 44:1680-1685.

21. Korsinczky M, Fischer K, Chen N, Baker J, Rieckmann K, Cheng Q: Sulfadoxine resistance in Plasmodium vivax is associated with a specific amino acid in dihydropteroate synthase at the putative sulfadoxinebinding site. Antimicrob Agents Chemother 2004, 48:2214-2222.

22. Jima D, Tesfaye G, Medhin A, Kebede A, Argaw D, Babaniyi O: Safety and efficacy of artemether-lumefantrine in the treatment of uncomplicated falciparum malaria in Ethiopia. East Afr Med J 2005, 82:387-390.

23. Aponte JJ, Schellenberg D, Egan A, Breckenridge A, Carneiro I, Critchley J, Danquah I, Dodoo A, Kobbe R, Lell B, May J, Premji Z, Sanz S, Sevene E, Soulaymani-Becheikh R, Winstanley P, Adjei S, Anemana $S$, Chandramohan D, Issifou S, Mockenhaupt F, Owusu-Agyei S, Greenwood B, Grobusch MP, Kremsner PG, Macete E, Mshinda H, Newman RD, Slutsker L, Tanner M, Alonso P, Menendez C: Efficacy and safety of intermittent preventive treatment with sulfadoxine-pyrimethamine for malaria in African infants: a pooled analysis of six randomised, placebo-controlled trials. Lancet 2009, 374:1533-1542.

24. Wongsrichanalai $\mathrm{C}$, Pickard AL, Wernsdorfer WH, Meshnick SR: Epidemiology of drug-resistant malaria. Lancet Infect Dis 2002, 2:209-218.

25. Contreras CE, Cortese JF, Caraballo A, Plowe CV: Genetics of drug-resistant Plasmodium falciparum malaria in the Venezuelan state of Bolivar. Am J Trop Med Hyg 2002, 67:400-405.

26. Nagesha HS, Din S, Casey GJ, Susanti Al, Fryauff DJ, Reeder JC, Cowman AF: Mutations in the pfmdr1, dhfr and dhps genes of Plasmodium falciparum are associated with in-vivo drug resistance in West Papua, Indonesia. Trans R Soc Trop Med Hyg 2001, 95:43-49.

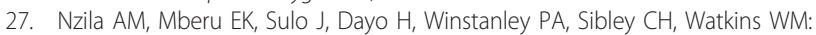
Towards an understanding of the mechanism of pyrimethaminesulfadoxine resistance in Plasmodium falciparum: genotyping of dihydrofolate reductase and dihydropteroate synthase of Kenyan parasites. Antimicrob Agents Chemother 2000, 44:991-996.

28. Schmider N, Peyerl-Hoffmann G, Restrepo M, Jelinek T: Short communication: point mutations in the dihydrofolate reductase and dihydropteroate synthase genes of Plasmodium falciparum isolates from Colombia. Trop Med Int Health 2003, 8:129-132.

29. Young MD, Burgess RW: Pyrimethamine resistance in Plasmodium vivax malaria. Bull World Health Organ 1959, 20:27-36.

30. Zakeri S, Motmaen SR, Afsharpad M, Djadid ND: Molecular characterization of antifolates resistance-associated genes, (dhfr and dhps) in Plasmodium vivax isolates from the Middle East. Malar J 2009, 8:20.

31. Auliff A, Wilson DW, Russell B, Gao Q, Chen N, Anh IN, Maguire J, Bell D, O'Neil MT, Cheng Q: Amino acid mutations in Plasmodium vivax DHFR and DHPS from several geographical regions and susceptibility to antifolate drugs. Am J Trop Med Hyg 2006, 75:617-621.

32. Rungsihirunrat $K$, Na-Bangchang K, Hawkins VN, Mungthin M, Sibley $C H$ : Sensitivity to antifolates and genetic analysis of Plasmodium vivax isolates from Thailand. Am J Trop Med Hyg 2007, 76:1057-1065.

33. Khatoon L, Baliraine FN, Bonizzoni M, Malik SA, Yan G: Prevalence of antimalarial drug resistance mutations in Plasmodium vivax and $P$. falciparum from a malaria-endemic area of Pakistan. Am J Trop Med Hyg 2009, 81:525-528.
34. Kaur S, Prajapati SK, Kalyanaraman K, Mohmmed A, Joshi H, Chauhan VS: Plasmodium vivax dihydrofolate reductase point mutations from the Indian subcontinent. Acta Trop 2006, 97:174-180.

35. Hawkins VN, Joshi $H$, Rungsihirunrat $K$, Na-Bangchang $K$, Sibley $C H$ : Antifolates can have a role in the treatment of Plasmodium vivax. Trends Parasitol 2007, 23:213-222.

36. Tjitra E, Baker J, Suprianto S, Cheng Q, Anstey NM: Therapeutic efficacies of artesunate-sulfadoxine-pyrimethamine and chloroquine-sulfadoxinepyrimethamine in vivax malaria pilot studies: relationship to Plasmodium vivax dhfr mutations. Antimicrob Agents Chemother 2002, 46:3947-3953.

37. Zakeri S, Afsharpad M, Ghasemi F, Raeisi A, Safi N, Butt W, Atta H, Djadid ND: Molecular surveillance of Plasmodium vivax dhfr and dhps mutations in isolates from Afghanistan. Malar J 2010, 9:75.

38. Rubio JM, Benito A, Roche J, Berzosa PJ, Garcia ML, Mico M, Edu M, Alvar J: Semi-nested, multiplex polymerase chain reaction for detection of human malaria parasites and evidence of Plasmodium vivax infection in Equatorial Guinea. Am J Trop Med Hyg 1999, 60:183-187.

39. 2002 PCR-ASRA Protocols for Plasmodium falciparum Drug-Resistance Mutation Analysis. [http://medschool.umaryland.edu/CVD/2002_pcr_asra. asp].

40. Dippmann AK, Bienzle U, Harms G, Mockenhaupt FP: pfmdr1 mutations in imported African Plasmodium falciparum isolates. Trans $R$ Soc Trop Med Hyg 2008, 102:1148-1150.

41. Newcombe RG: Two-sided confidence intervals for the single proportion: comparison of seven methods. Stat Med 1998, 17:857-872.

42. Kebede F, Taffa N, Tedla T: An in-vivo study of falciparum malaria sensitivity to Chloroquine in unstable malaria endemic area of central Ethiopia. Ethiop Med J 1999, 37:97-109.

43. Schunk M, Kumma WP, Miranda IB, Osman ME, Roewer S, Alano A, Loscher T, Bienzle U, Mockenhaupt FP: High prevalence of drug-resistance mutations in Plasmodium falciparum and Plasmodium vivax in southern Ethiopia. Malar J 2006, 5:54.

44. Karunamoorthi K, Bekele M: Prevalence of malaria from peripheral blood smears examination: a 1-year retrospective study from the Serbo Health Center, Kersa Woreda, Ethiopia. J Infect Public Health 2009, 2:171-176.

45. Djimde A, Doumbo OK, Cortese JF, Kayentao K, Doumbo S, Diourte Y, Dicko A, Su XZ, Nomura T, Fidock DA, Wellems TE, Plowe CV, Coulibaly D: A molecular marker for chloroquine-resistant falciparum malaria. N Engl J Med 2001, 344:257-263.

46. Mockenhaupt FP, Ehrhardt S, Eggelte TA, Agana-Nsiire P, Stollberg K, Mathieu A, Markert M, Otchwemah RN, Bienzle U: Chloroquine-treatment failure in northern Ghana: roles of pfcrt T76 and pfmdr1 Y86. Ann Trop Med Parasitol 2005, 99:723-732.

\section{doi:10.1186/1475-2875-10-214}

Cite this article as: Mula et al:: Detection of high levels of mutations involved in anti-malarial drug resistance in Plasmodium falciparum and Plasmodium vivax at a rural hospital in southern Ethiopia. Malaria Journal 2011 10:214.

\section{Submit your next manuscript to BioMed Central and take full advantage of:}

- Convenient online submission

- Thorough peer review

- No space constraints or color figure charges

- Immediate publication on acceptance

- Inclusion in PubMed, CAS, Scopus and Google Scholar

- Research which is freely available for redistribution

Submit your manuscript at www.biomedcentral.com/submit
C Biomed Central 